\title{
ROBOT-BASED SURFACE FINISHING OF FORMING DIES CONCERNING DIFFERENT PATH STRATEGIES
}

\author{
S. Bay ${ }^{1 *}$, P. Bossong ${ }^{1}$, M. Weigold ${ }^{1}$ \\ ${ }^{1}$ Technical University of Darmstadt (TUDa), Institute of Production Management, Technology and \\ Machine Tools (PTW); Otto-Berndt-Straße 2, 64287 Darmstadt, Germany \\ *Corresponding author; e-mail: stephan.bay@tu-darmstadt.de
}

\begin{abstract}
The production of shape accurate forming dies in the toolmaking industry requires a high degree of manual labour. To achieve a higher level of automation, machine-based approaches are necessary. Therefore, robot-based milling experiments of difficult-to-machine cold work steel 1.2379 are carried out. This paper presents an experimental study of different path strategies using an industrial robot to mill example geometries of a forming die. Starting with preliminary tests to determine the best possible process and machining parameters, a conventional machining process strategy follows as a reference. Based on the findings two different strategies are derived: additional tool inclination and the avoidance of circular movements that are eventually joined and regarded in combination. Four different path strategies are carried out to determine the influence on form deviation in a CNC-like multi-step material removal process. Results are analysed and discussed regarding geometric fidelity and cutting moments of the example geometries.
\end{abstract}

Keywords:

Industrial Robots; Milling; Machining Strategies; Forming Dies

\section{INTRODUCTION}

In the automotive industry, industrial robots are widely used for handling and welding applications [IFR 2020] where high precision and accuracy are not required. When comparing machine tools and industrial robots of the same working space size, industrial robots are cheaper regarding their investment costs [Schmid 2017]. This is particularly important in the toolmaking industry as the machining of forming tools requires a large working space. The high flexibility of industrial robots regarding different end effectors combined with their large working space results in a boost in efficiency. However, using industrial robots in milling applications poses high process forces on the robot. Due to the robot's low stiffness, this leads to large displacements of the milling tool and consequently impairs surface accuracy of the machined part. Therefore, optimised milling strategies that reduce form deviations are needed and addressed in this paper.

Starting with an overview of recent literature, chapter 2 summarises the state of the art concerning toolpath and pose-dependent influences in robotic machining applications. Based on the experimental setup, which is described in chapter 3 , preliminary experiments and the machining of a reference workpiece are conducted in order to derive optimised toolpath strategies in chapter 4 . The results of the experiments regarding the new toolpath strategies are presented in chapter 5 , while a conclusion and recommended actions are given in chapter 6 .

\section{STATE OF THE ART}

[Bisu 2011] conduct a position-dependent modal analysis to identify the influence of different working positions on the dynamic behaviour of a KUKA KR 240-2. Their results indicate that the robot becomes stiffer in $\mathrm{x}$-direction and more compliant in $z$ - and $y$-direction the further the tool centre point (TCP) is located away from the robot base frame, even though a possible explanation for this observed pattern is not given.

By investigating the influence of the tool orientation on surface quality, [Coelho 2011] show that best results with an ABB IRB 240 can be achieved, when the tool is parallel to the world's z-axis. They attribute that to the higher cartesian stiffness in this direction. Nevertheless, lower surface roughness can be achieved when inclining the tool, but the lower stiffness in this position causes the toolpaths to deviate more.

By experimentally analysing the eigenfrequencies of an ABB IRB 6660 for ten different TCP positions, [Mejri 2016] find that the robot is less compliant along the world's y-axis than along the other axes. Their results further suggest that the robot's dynamic behaviour is invariant to the TCP MM Science Journal | 2021 | NOVEMBER - Special Issue on HSM2021 
position when choosing a toolpath that causes the major cutting force to be parallel to the wrist axis. Hence, the feed direction is an important factor for stability.

Similar to [Mejri 2016], [Li 2017] conduct a modal analysis and aim at identifying how the toolpath and the clamping position of the workpiece influence the machining results. The stiffness along the $x$-axis has been identified to be more rigid. Moreover, a clamping position centred right in front of the robot has been found to be advantageous.

[Cordes 2017] use the joint stiffness and reversal error to predict the path deviation of robot milling processes. By milling circular contours and applying the developed joint stiffness and hysteresis model, the roundness is improved by $31 \%$. Furthermore, they discover that no significant backlash occurs at the zero crossing of joint 1.

With the help of two different reference geometries that require different feed directions, an analysis of the pose and feed direction influence on the milling process is conducted by [Karim 2018b]. Those geometries are milled in nine different poses showing that milling along the world's $y$-axis should be preferred to milling in x-direction. Depending on the objective, the optimal pose varies: While a pose closer to the robot's base frame leads to the best surface quality, a pose further away leads to smaller process forces.

In line with previous findings, [Karim 2018a] find that chatter is strongly dependent on the feed direction. Even though they analyse twelve different feed directions with varying spindle speed, they are not able to identify the reason for their finding that the chatter frequency is highly dependent on the feed direction, whereas the chatter amplitude depends on spindle speed.

[Hao 2019] analyses the pose-dependent stiffness of an industrial robot. For their modal analyses, they propose a new approach, based on moving and weighted average calculation, to merge low and high frequency signals from two sensors. They find that the dynamic stiffness of the robot increases, the further the TCP is located away from the base frame along the world's $x$-axis. The authors therefore conclude that in this case high process forces should be taken up in $x$-direction. Through milling tests, they validate their findings, while it remains unclear which process parameters have been used throughout those experiments.

\section{EXPERIMENTAL SETUP}

\subsection{Robotic system}

The experiments are carried out using a $27 \mathrm{~kW} \mathrm{AC-spindle}$ from Franz Kessler $\mathrm{GmbH}$ (weighing $108 \mathrm{~kg}$ ) mounted to a KUKA KR 300 R2500 ultra, six-axis, articulated robot. As the robot is able to handle a significantly higher payload, up to $300 \mathrm{~kg}$, it can be considered to be oversized regarding the static payload. This ensures higher rigidity of the robot structure and allows high acceleration profiles of the TCP.

For the force and torque measuring, the sensory tool holder system SPIKE (pro-micron $\mathrm{GmbH}$, Germany) is used.

\subsection{Milling tools}

Each machining step is conducted with the same type of tool. For roughing steps, an ordinary end-mill with an edge radius of $1 \mathrm{~mm}$ is used, whereas finishing steps are conducted with a ball-nose end-mill according to Tab. 1. The used tools are Chip Surfer from Ingersoll.
Tab. 1: Milling tools used for the experiments

\begin{tabular}{cccccc}
\hline Usage & $\begin{array}{c}\text { Mill } \\
\text { type }\end{array}$ & $\begin{array}{c}\text { Dia- } \\
\text { meter }\end{array}$ & $\begin{array}{c}\text { Cutting } \\
\text { edges }\end{array}$ & $\begin{array}{c}\text { Flanging } \\
\text { radius }\end{array}$ & Quality \\
\hline Roughing & End & $10 \mathrm{~mm}$ & 4 & $1 \mathrm{~mm}$ & IN2005 \\
& & & & $6 \mathrm{~mm}$ & IN2005 \\
\hline
\end{tabular}

\subsection{Workpiece and geometry}

The geometry of the workpiece is based on a feature abstraction of a complex forming tool. A finished robot machined workpiece is shown in Fig. 1.

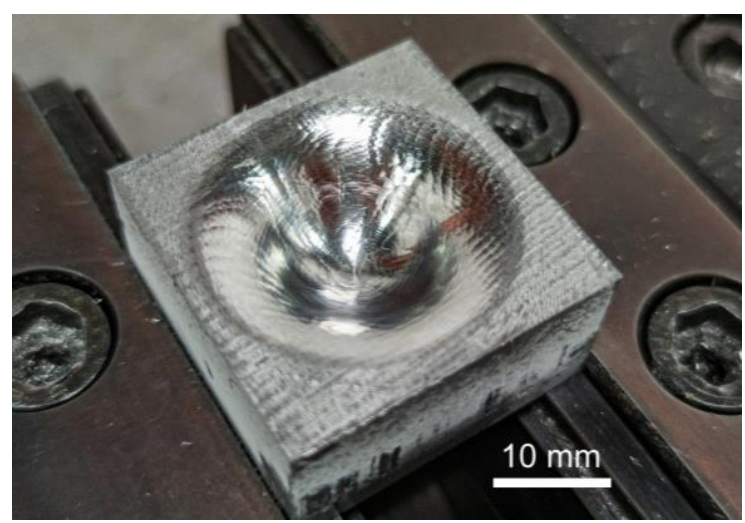

Fig. 1: Robot machined workpiece with spherical cavity

A spherical cavity with rounded borders is selected to reveal occurring milling process deviations in as many directions in space as possible.

It has the dimensions $40 \mathrm{~mm} \times 40 \mathrm{~mm} \times 25 \mathrm{~mm}$ and consists of 1.2379 cold work steel, which is commonly used in the die making industry.

\subsection{Machining steps}

Irrespective of the chosen path strategy, the whole machining process consists of one roughing step, followed by three finishing steps. The tool paths are generated using the software Mastercam. Robotmaster is used for the conversion into robot code.

Tab. 2: Overview of the machining steps

\begin{tabular}{lcccc}
\hline & Roughing & Pre finish & Finish & Fine finish \\
\hline $\begin{array}{l}\text { Stock to } \\
\text { leave }\end{array}$ & $\begin{array}{c}\text { Min. } \\
0.6 \mathrm{~mm}\end{array}$ & $0.6 \mathrm{~mm}$ & $0.2 \mathrm{~mm}$ & $0.0 \mathrm{~mm}$ \\
$\begin{array}{l}\text { Machining } \\
\text { areas (top } \\
\text { view) }\end{array}$ & & & & \\
\hline
\end{tabular}

After the roughing process, which covers the whole surface, the first pre-finishing process mills the geometry's cavity and omits its corners as the sketch in Tab. 2 shows. This smoothens out the surface after the roughing process and leaves $0.6 \mathrm{~mm}$ on stock for the following finishing steps. The descending offset to the target geometry results in a descending value for the maximum depth of cut. 


\section{TOOL PATH STRATEGIES}

\subsection{Overview}

The experiments follow the sequence shown in the flowchart in Fig. 2 and are designed as follows: By conducting preliminary milling experiments, it is determined which robot pose and clamping position of the workpiece lead to best surface accuracy (section 4.2). In order to identify how path strategies, process forces and surface accuracy are interrelated with each other, a conventional milling strategy is carried out (section 4.3). Based on the machining results of this conventional strategy, new and optimised strategies are derived, which examine tool inclination and circular movement avoidance (sections 4.4 and 4.5). By combining those new strategies, a third optimised tool path strategy is investigated (section 4.6).

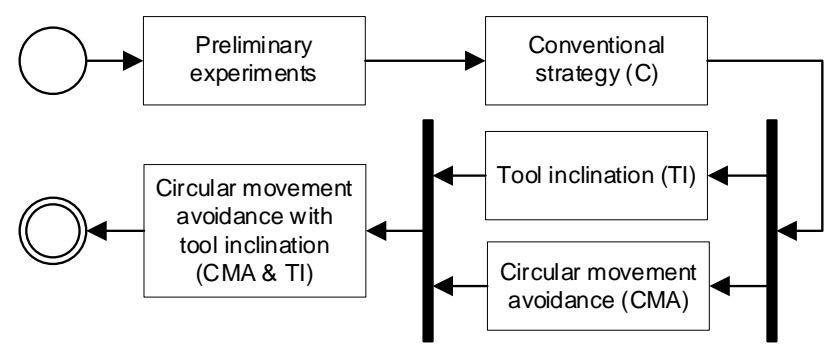

Fig. 2: Activity diagram of the experiments

After the most promising process parameters have been identified during the preliminary experiments, they remain unchanged according to chapter 3 in order to only change the movement path of the tool to ensure comparability between the strategies. In total, this paper describes and evaluates four different toolpath strategies.

\subsection{Preliminary experiments}

Different preliminary experiments regarding the robot pose, milling parameters and milling direction were carried out to determine the most advantageous robot pose and clamping position. The design of the preliminary experiments has been structured in a way that multiple positions, poses and milling parameters have been tried out for a reference workpiece. Subsequently parameter combinations which led to the best surface accuracy, lowest surface roughness and fastest machining time have been identified.

\section{Robot pose}

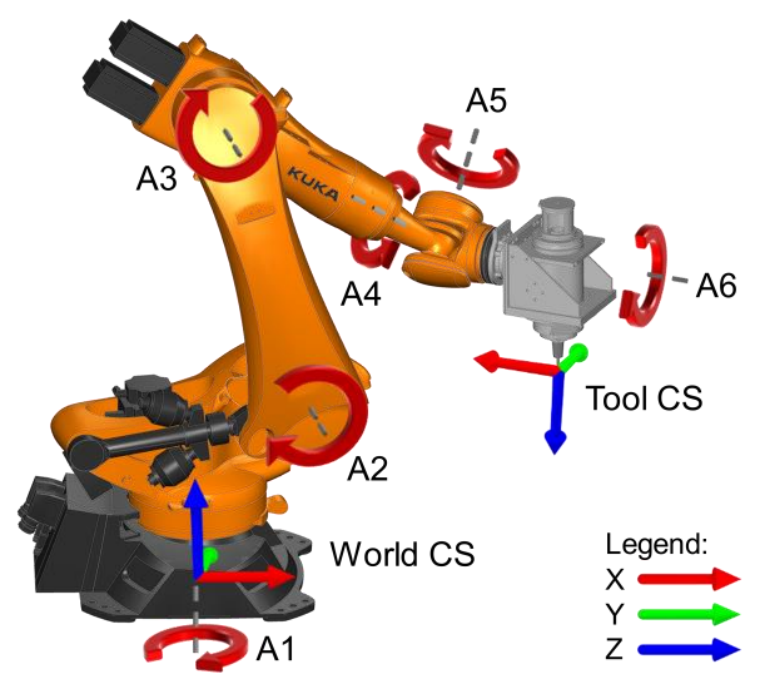

Fig. 3: Robot pose, coordinate systems (CS) and work position of the TCP during the experiments
By using a robot pose with a folded wrist (axes 4 to 6 ) where axis 5 is not in the XY-plane of the tool CS as depicted in Fig. 3, preliminary tests have shown that the forces from the milling process can be better absorbed by the robot axis motors compared to a non-folded pose. This results in less form deviations.

To ensure consistency, the same angulation of $155^{\circ}$ between the robot's $x$-axis and the tool's $x$-axis is maintained throughout the experiments.

\section{Milling parameters}

Starting with the milling parameters which are recommended by the tool manufacturer, a statistical design of experiments has been used to determine the following milling parameters for the roughing (Tab. 3 ) and finishing (Tab. 4) processes. To ensure that the deviation of the cutting tool is less than the offset to the resulting part, more conservative parameters regarding the depth and width of the cut are chosen compared to the capabilities of the robot.

Tab. 3: Milling parameters for the roughing process

\begin{tabular}{lr}
\hline Parameters for roughing & \\
\hline Revolutions per minute $(\mathrm{n})$ & $4774 \mathrm{rpm}$ \\
Cutting speed $\left(\mathrm{v}_{\mathrm{c}}\right)$ & $150 \mathrm{~m} / \mathrm{min}$ \\
Tooth feed $\left(\mathrm{f}_{\mathrm{z}}\right)$ & $0.012 \mathrm{~mm} /$ tooth \\
Feed rate $\left(\mathrm{V}_{\mathrm{f}}\right)$ & $230 \mathrm{~mm} / \mathrm{min}$ \\
Max. depth of cut $\left(\mathrm{a}_{\mathrm{p}}\right)$ & $0.6 \mathrm{~mm}$ \\
Max. width of cut $\left(\mathrm{a}_{\mathrm{e}}\right)$ & $2.1 \mathrm{~mm}$ \\
\hline
\end{tabular}

Tab. 4: Milling parameters for the finishing processes

\begin{tabular}{lr}
\hline Parameters for finishing \\
\hline Revolutions per minute $(\mathrm{n})$ & $11459 \mathrm{rpm}$ \\
Cutting speed $\left(\mathrm{V}_{\mathrm{c}}\right)$ & $432 \mathrm{~m} / \mathrm{min}$ \\
Tooth feed $\left(\mathrm{f}_{\mathrm{z}}\right)$ & $0.04 \mathrm{~mm} /$ tooth \\
Feed rate $\left(\mathrm{V}_{\mathrm{f}}\right)$ & $916 \mathrm{~mm} / \mathrm{min}$ \\
Max. depth of cut $\left(\mathrm{ap}_{\mathrm{p}}\right)$ & $0.2 \mathrm{~mm}$ \\
Max. width of cut $\left(\mathrm{a}_{\mathrm{e}}\right)$ & $0.5 \mathrm{~mm}$ \\
\hline
\end{tabular}

\section{Workpiece clamping position and orientation}

The clamping position of the workpiece within the robot's working space influences the overall robot pose and therefore the stiffness of the system. From preliminary experiments with nine different clamping positions in the robot's working space, the position right in front of the robot (see Tab. 5) has resulted in lowest surface deviations. This is in line with previous results by [Li 2017] and [Cordes 2017] who find that the comparably low gear stiffness of the robot makes it necessary to place the workpiece as close to the robot as possible in order to reduce the lever arm.

Tab. 5: Approximate workpiece position and orientation regarding the world coordinate system of the robot

\begin{tabular}{cccccc}
\hline $\mathbf{X}$ & $\mathbf{Y}$ & $\mathbf{Z}$ & $\mathbf{A}$ & $\mathbf{B}$ & $\mathbf{C}$ \\
\hline $1260 \mathrm{~mm}$ & $-50 \mathrm{~mm}$ & $810 \mathrm{~mm}$ & $1.0^{\circ}$ & $-0.5^{\circ}$ & $0.2^{\circ}$ \\
\hline
\end{tabular}
MM Science Journal | 2021 | NOVEMBER - Special Issue on HSM2021 
Additionally, for the roughing process the feed direction is oriented in the direction of the negative $x$-axis and positive $y$-axis of the world CS which is along the same direction as the positive $x$-axis of the tool CS in Fig. 3. Compared to the other directions ( $45^{\circ}$ sections), this pulling movement results in the lowest average surface roughness of $R_{z}=$ $10,01 \mu \mathrm{m}$ during the preliminary experiments.

\subsection{Conventional strategy (C)}

In order to generate a reference dataset from which optimised strategies can be derived and compared to, a too path strategy is carried out which could also be used on ordinary 3-axis machine tools. Therefore, each of the three finishing steps is characterised by paths that are guided by the circular cavity as shown in Fig. 4.

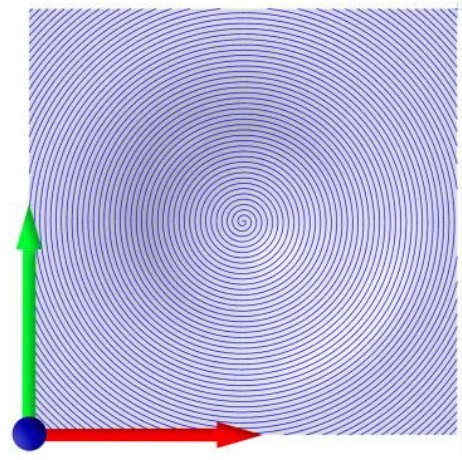

Fig. 4: Path planning for the conventional strategy

The geometry's plane areas are machined through spiral cuts which are interrupted by the workpiece's edges. The cavity is machined through continuous spiral movements from the cavity's upper border downwards. Tool orientation is maintained parallel to the world coordinate system's zaxis throughout all machining steps making this strategy also feasible for conventional machine tools.

The form deviation of the final machining result, which has been generated with the conventional strategy is shown in Fig. 5.

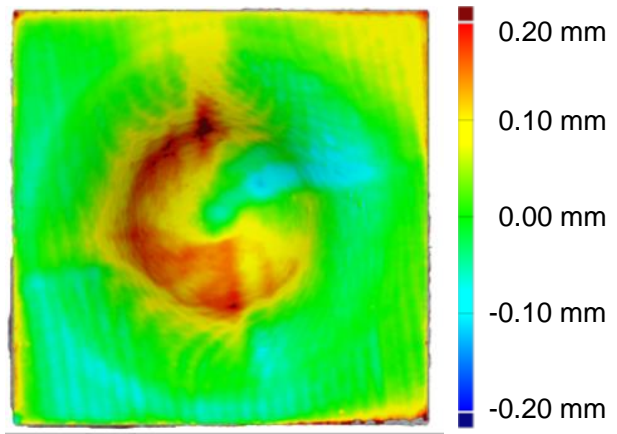

Fig. 5: Form deviation (false colour) of the conventional strategy

As the colour scale indicates, this strategy produces deviations smaller than $+/-100 \mu \mathrm{m}$ in the transition areas between the workpiece's plane areas and its cavity. However, the steep parts of the cavity exhibit significant surface deviations of more than $200 \mu \mathrm{m}$, which violates the tolerances that are required in forming tools manufacturing. The surface deviation measures are exhibited in Tab. 6 and serve as benchmark which the other optimised strategies are compared to.
Tab. 6: Measured deviations of the conventional strategy

\begin{tabular}{ccccc}
\hline Min. & Max. & Abs. Mean & Median & $\boldsymbol{\sigma}$ \\
\hline$-147 \mu \mathrm{m}$ & $239 \mu \mathrm{m}$ & $54 \mu \mathrm{m}$ & $3 \mu \mathrm{m}$ & $69 \mu \mathrm{m}$ \\
\hline
\end{tabular}

\subsection{Tool inclination strategy (TI)}

Looking at the process forces and moments during the prefinishing step of the conventional strategy, it can be seen that the bending moment in Fig. 6 appears to oscillate stronger in the red marked area between $160 \mathrm{~s}$ and $200 \mathrm{~s}$. An analysis of variance for the signal underlines this finding: The bending moment's variance in this area is $6 \%$ higher than the variance prior to $160 \mathrm{~s}$. In this area, the milling takes place at the steepest part of the cavity. Due to the geometry of the ball-nose end-mill, larger parts of the tool are engaged in steep areas. The stronger variance of the bending moment could therefore be an indicator that the tool touches the workpiece surface more irregularly.

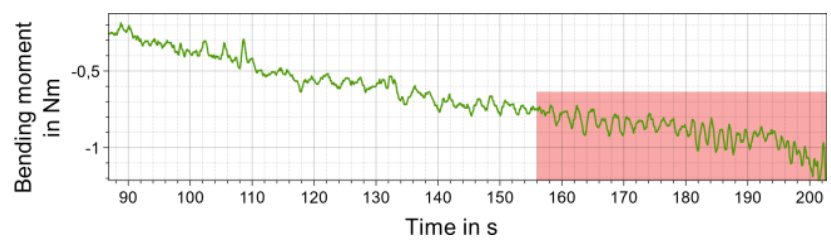

Fig. 6: Bending moment during the conventional strategy

Based on these findings, it is proposed for the tool inclination strategy: A dynamic inclination of the tool leads to a more consistent tool contact point or contact area, respectively, and consequently to more accurate surfaces. Therefore, the conventional strategy is slightly altered, using cuts for the plane areas that are parallel to the geometry's edges, and an inclined spiral motion for the cavity as seen in Fig. 7. The inclination follows the workpiece surface but has to be restricted to a maximum of $5^{\circ}$ about the world coordinate system's $z$-axis in order to generate feasible robot poses.

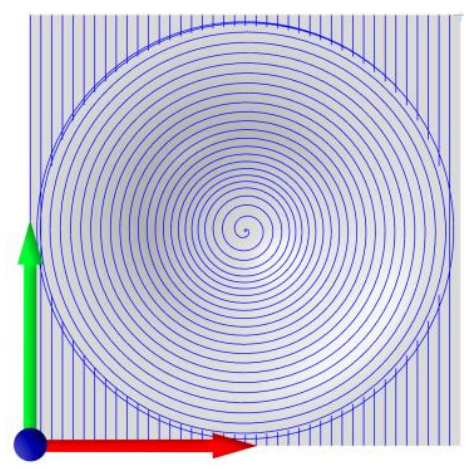

Fig. 7: Path planning for the tool inclination strategy

\subsection{Circular movement avoidance strategy (CMA)}

Another valuable insight from the conventional strategy results can be gained through the signed deviation graph in Fig. 8. The surface deviation progression throughout the counter clockwise helical movements that start with large radii at the top of the workpiece are shown. 


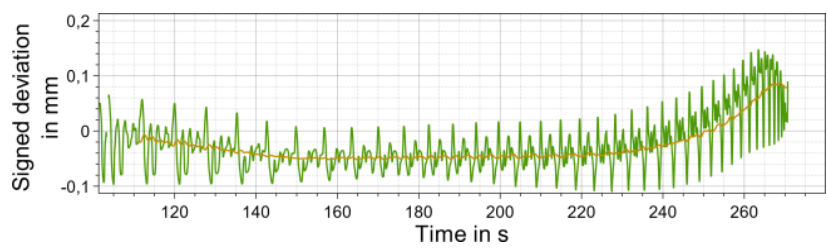

Fig. 8: Signed deviation graph (green: unfiltered, yellow: moving average (600))

With increasing depth (i.e., advancing in negative $z$ direction), the circles become smaller. This can also be seen from the unfiltered signed deviation graph: While it takes approximately $8 \mathrm{~s}$ for the tool to travel along the first circle ( $112 \mathrm{~s}$ to $120 \mathrm{~s}$ ), it takes only $0.4 \mathrm{~s}$ to finish the last circle on the ground of the workpiece's cavity. This leads to two effects:

1. Smaller circles lead to a positive surface deviation as the growing average deviation graph in Fig. 8 visualises. This indicates that the robot has difficulties to accurately follow those small helical movements causing disadvantageous surface deviations and leading to the hypothesis that surface accuracy can be increased by avoiding circular movements with a small radius.

2. The narrow circles lead to a low relative speed between the end-mill and the workpiece. Especially due to the ball-nose geometry, only the end-mill's tip is engaged in the material. This leads to cutting speeds that are very small since the end-mill's angular velocity is zero at its centre point. Therefore, in line with previous findings in section 4.4 it is expected that an optimised tool inclination could mitigate this drawback because it leads to a more consistent tool contact area.

By moving the tool from the cavity's bottom towards the top, CMA avoids circular movements as shown in Fig. 9. The path strategy for the plane areas remains similar to the one of $\mathrm{Tl}$.

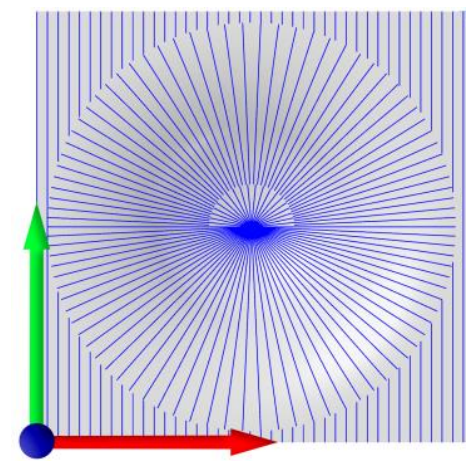

Fig. 9: Path planning for the circular movement avoidance strategy

\subsection{Circular movement avoidance with tool inclination strategy (CMA and TI)}

By combining the two strategies CMA and TI, the circular movement avoidance with tool inclination strategy is developed as exhibited in Fig. 10.

By using toolpaths that are parallel to the workpiece's edges, circular movements are avoided, and the feed direction follows approximately the world coordinate system's y-axis which has been identified to be advantageous by [Karim 2018b]. Furthermore, the tool is inclined by up to a maximum of $5^{\circ}$ about the world's z-axis. This restriction is required to generate feasible robot poses. Consequently, small circular movements are avoided while tool inclination is improved.

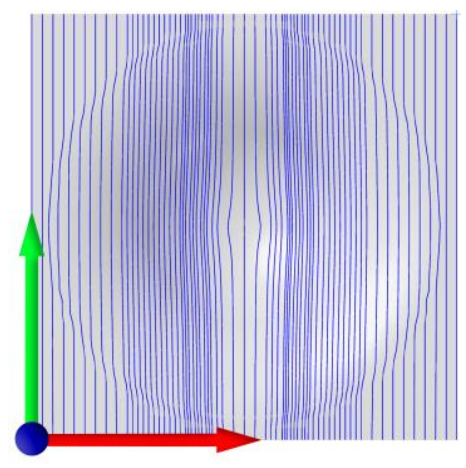

Fig. 10: Path planning for the circular movement avoidance with tool inclination strategy

\section{EXPERIMENTAL RESULTS AND DISCUSSION}

After the new toolpath strategies have been hypothesised based on the conventional strategy results, those new strategies are machined and analysed. Since the results of the preliminary experiments show repeatable surface deviations, one example of each path strategy is conducted and discussed.

\subsection{Results and discussion of the tool inclination strategy (TI)}

Looking on the surface deviations visualisation of Fig. 11 reveals the following findings:

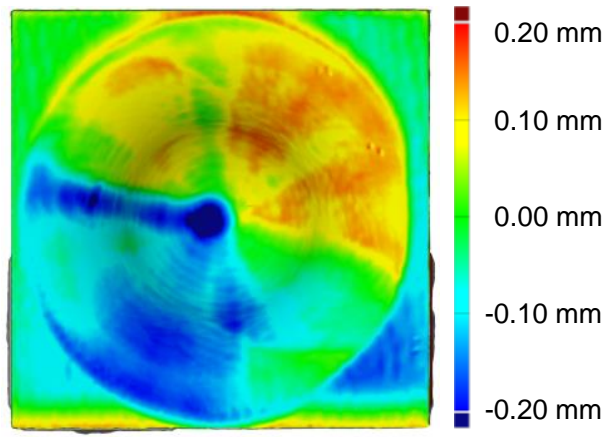

Fig. 11: Form deviation (false colour) of the tool inclination strategy

The plane surface areas, which are machined in positive ydirection, exhibit form deviations up to $140 \mu \mathrm{m}$ on the lower edge than on the upper one. Especially near the workpiece's $x$-axis, excessive material remains. This could be due to the arc-shaped plunge movements of the tool since a small discrepancy between the actual and the expected workpiece dimensions can occur. This leads to a plunge movement that lets the tool engage in the material too late, leaving excessive material on the edge. Moreover, the tool appears to plunge too deep into the material as the negative surface deviations especially in the bottom right corner show. Therefore, it is important to ensure that the plunge movement is tangential to the surface and considers dimension tolerances.

The workpiece's cavity exhibits a deviation pattern that seems to be reflected on the cavity's bottom point: Areas of positive deviation on one side occur on the other side with a negative deviation that is about the same absolute value. This could be due to the strong movements of multiple axes which constantly change the position- and directiondependent stiffness of the robot or due to tool deflection that pushes the tool towards or away from the workpiece, respectively. The graphs in Fig. 12 compare the bending moment between $\mathrm{TI}$ and the conventional strategy. As both 
graphs exhibit, the bending moment oscillates periodically throughout the circular movements. The conventional strategy exhibits a slightly more balanced oscillation pattern, but a drift can be observed that lets the bending moment become stronger, the deeper the tool advances downwards the cavity. As a bending moment drift does not occur in $\mathrm{TI}$, it seems more probable that the deviations have been caused by the strong axis movements and not by tool deflection.
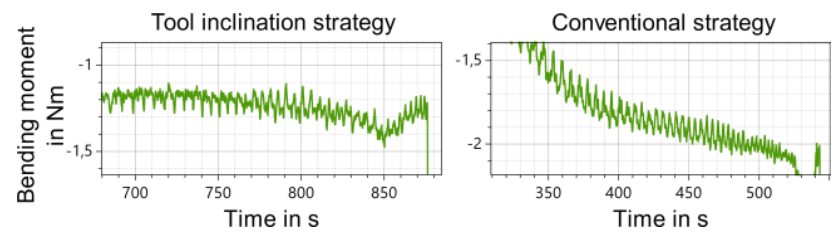

Fig. 12: Bending moments (moving average (1000)) of strategies $\mathrm{TI}$ and $\mathrm{C}$

Compared to the conventional strategy, only the maximum positive form deviation of the five surface measures for TI (Tab. 7) results in a better figure. This proves that TI does not lead to better results than the conventional strategy.

Tab. 7: Measured deviations of the tool inclination strategy

\begin{tabular}{ccccc}
\hline Min. & Max. & Abs. Mean & Median & $\boldsymbol{\sigma}$ \\
\hline$-264 \mu \mathrm{m}$ & $178 \mu \mathrm{m}$ & $82 \mu \mathrm{m}$ & $-12 \mu \mathrm{m}$ & $101 \mu \mathrm{m}$ \\
\hline
\end{tabular}

\subsection{Results and discussion of the circular movement avoidance strategy (CMA)}

The circular movement avoidance strategy focusses on the avoidance of circular movements. As the surface deviations in Fig. 13 show, the upper edge of the workpiece and the lower edge of the cavity are again characterised by excessive material that has not been taken off, probably due to the arc-shaped retract movement that does not consider any dimension tolerances of the raw stock. In addition, it is noticeable that the cavity exhibits an elliptical area in which too much material has been taken off. This area is not centred in the middle of the workpiece because the right slope of the cavity has less form deviation than the left slope.

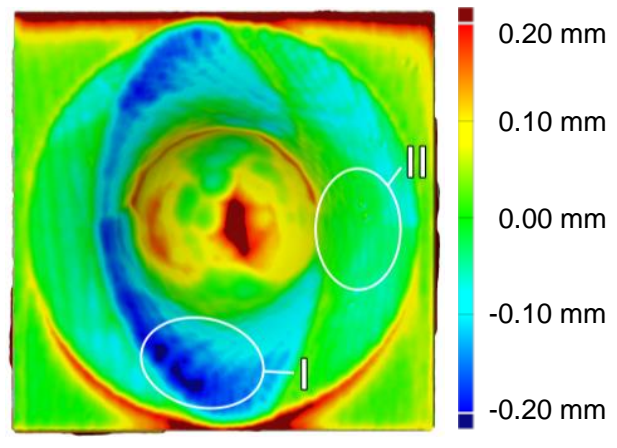

Fig. 13: Form deviation (false colour) of the circular movement avoidance strategy

There could be multiple reasons for this pattern:

1. The tool could be deflected due to the steep slope of the cavity. However, the process forces do not provide any characteristic moment or tension patterns.

2. More likely there could be TCP velocity deviations that could cause strong surface deviations. But examining the exemplary velocities graph of Fig. 14 shows a velocity accuracy of $+/-0.0025 \mathrm{~m} / \mathrm{s}$ during the steep areas of the cavity. The velocity discrepancy spikes are caused by the rapid repositioning movement during which the tool is not engaged. Right after the repositioning movement, the tool plunges into the bottom of the cavity which causes the actual velocity to deviate from the desired velocity. This could be a possible explanation for the excessive material that remains on the cavity's bottom but is not able to explain the elliptical deviation pattern.

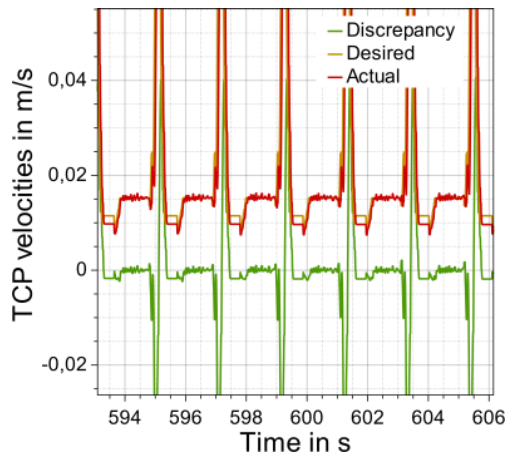

Fig. 14: Velocities of the TCP during the CMA strategy

3. As the graph in Fig. 15 exhibits, the tool orientation about axis B (i.e., rotation about the tool's $y$-axis), which should be constant at zero degrees, deviates while the tool is engaged.

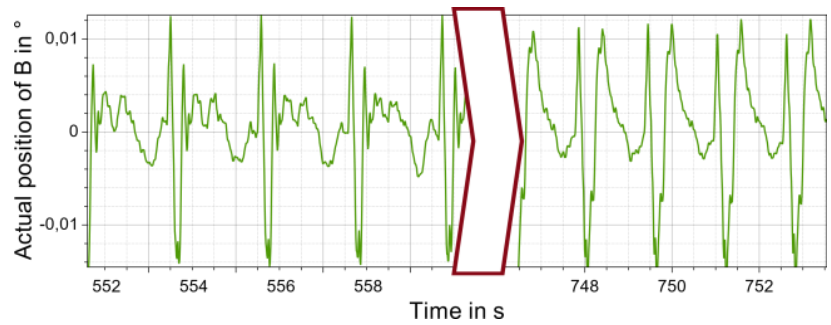

Fig. 15: Tool orientation about axis $B$

The graph is divided into two parts: The left part shows an excerpt from the tool orientation progression while area I (marked in the surface deviation picture in Fig. 13) is machined and the right side represents area II. The signed deviation graph (Fig. 16) is split accordingly.

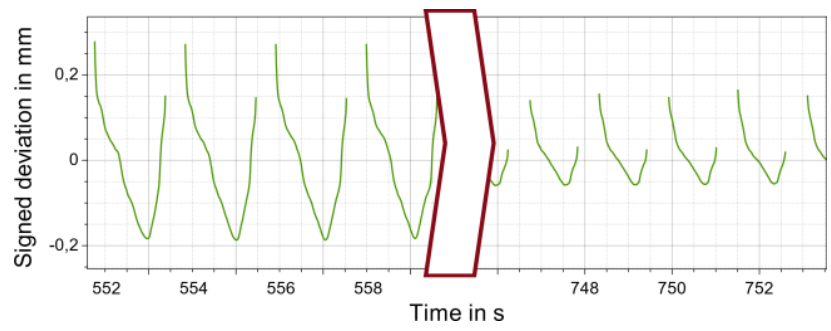

Fig. 16: Signed deviation graph for the circular movement avoidance strategy

It can be seen that a negative tool orientation about the Baxis is connected with the negative deviations that occur within the elliptical deviation area. Comparing the left and the right sides of the graphs reveal that surface deviations are stronger the more negative the maximum tool orientation deviation becomes. A possible reason could be that the robot performs a pushing movement in area II, whereas the opposite slope is machined with a pulling 
movement. This could lead to different robot behaviours due to the direction-dependent compliance of the robot. By starting the toolpath in the middle of the cavity, axis 1 rotates in negative direction for the pulling motion and in positive direction for the pushing motion. Since axis 1 shows hysteresis behaviour, which has an increasing influence the lower the cutting forces are, as demonstrated by [Cordes 2017] the change in the rotational movement direction of axis 1 combined with the low forces during the finishing process contributes to the surface deviation errors. Summarised, as the surface deviation measures in Tab. 8 show, CMA is also not able to achieve better measured deviations than the conventional strategy. Avoiding circular movements does not appear to have an advantageous impact on surface accuracy as a change of the toolpath causes tool deflections that dilute the expected enhancements through circular movement avoidance.

Tab. 8: Measured deviations of the circular movement avoidance strategy

\begin{tabular}{ccccc}
\hline Min. & Max. & Abs. Mean & Median & $\boldsymbol{\sigma}$ \\
\hline$-222 \mu \mathrm{m}$ & $281 \mu \mathrm{m}$ & $69 \mu \mathrm{m}$ & $6 \mu \mathrm{m}$ & $87 \mu \mathrm{m}$ \\
\hline
\end{tabular}

\subsection{Results and discussion of the circular movement avoidance with tool inclination strategy (CMA and TI)}

CMA in combination with TI examines both, the influence of a dynamic tool inclination and the avoidance of circular movements by replacing the circular movements that form the cavity by parallel movements that cover the whole surface.

The plunge movements along the workpiece edges remain similar to the ones in TI. This could be the reason why the surface deviations in Fig. 17 show a similar pattern on the lower edge where excessive material has remained on the workpiece.

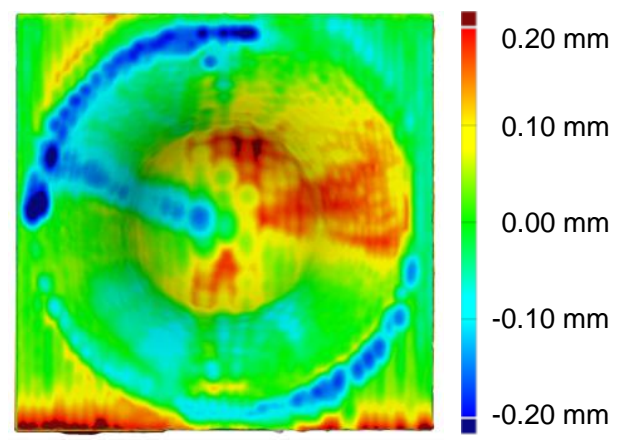

Fig. 17: Form deviation (false colour) of the strategies CMA and TI in combination

The deviation pattern is characterised by pits, especially along the cavity's circular corner, in which too much material has been removed. They can be better seen in Fig. 20 and are exemplarily highlighted with red arrows. Within the cavity, the pits are not as distinct as they are along its border. However, within the cavity, they predominantly occur on the bottom. Considering the graphs, that show the tool orientation's (Fig. 19) and the velocities' progression (Fig. 18), it is remarkable that the TCP velocity drops frequently, and the tool orientation changes rapidly.

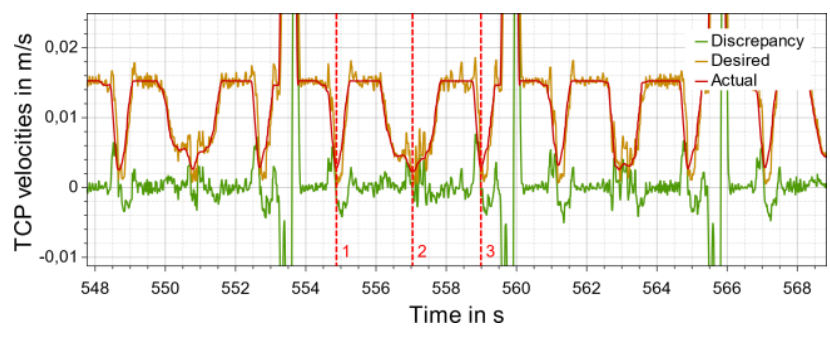

Fig. 18: Progression of the TCP velocities during the combination of CMA and TI

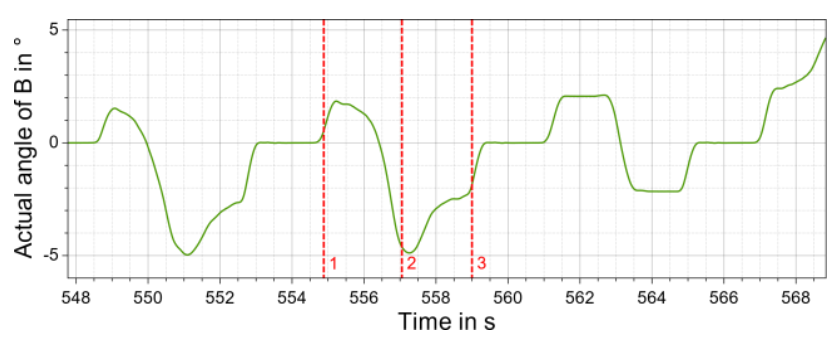

Fig. 19: Progression of the tool orientation angle $B$ during the combination of CMA and TI

The extraordinary high velocity peaks are caused by rapid repositioning movements once the tool has reached the edge of the workpiece. Between each rapid movement, three drops in the desired velocity can be identified which correspond to the numbered tool positions on the right side of Fig. 20 and the red dashed lines in Fig. 18 and Fig. 19: The first drop occurs when the tool passes the cavity's circular border, the second one can be found on the bottom of the cavity, and the third one is again located on the border of the cavity. The drops coincide with an oscillating actual velocity, causing a TCP velocity discrepancy. At all those three positions, pits occur. Moreover, the tool orientation about axis B changes rapidly (on the cavity's border, label 1 and 3 in Fig. 20) or is inverted (on the cavity's bottom, label 2 in Fig. 20).
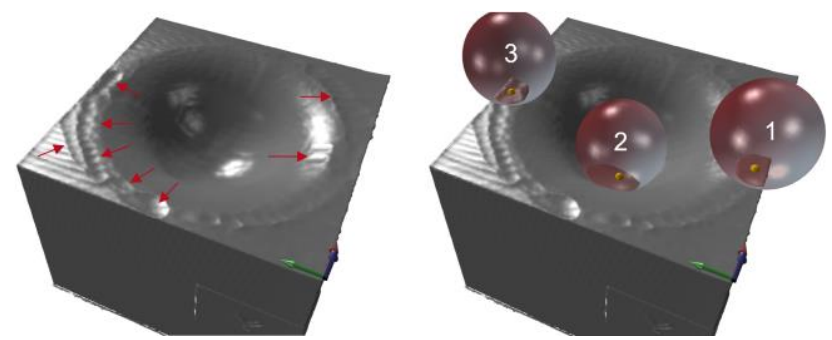

Fig. 20: Pits along the cavity's circular corner

The process forces, however, do not provide any explanatory power. Therefore, it can be concluded that changing the tool orientation while the TCP velocity is low leads to the disadvantageous surface results as seen in Fig. 17. For the toolpath planning strategy follows, if a change of tool orientation is required, this change should be carried out smoothly and without impacting TCP velocity.

Even though the surface measures for the CMA and TI strategy in Tab. 9 provide slightly better results than the measures for TI, both strategies are not able to compete with the conventional strategy. 
Tab. 9: Measured deviations of CMA and TI in combination

\begin{tabular}{ccccc}
\hline Min. & Max. & Abs. Mean & Median & $\boldsymbol{\sigma}$ \\
\hline$-252 \mu \mathrm{m}$ & $255 \mu \mathrm{m}$ & $65 \mu \mathrm{m}$ & $4 \mu \mathrm{m}$ & $80 \mu \mathrm{m}$ \\
\hline
\end{tabular}

Summarised, the preceding results show that a dynamic tool inclination in combination with parallel paths that avoid circular movements requires the robot to perform complex movements resulting in the concurrent movement of multiple axes and impacting TCP velocity. Both has a disadvantageous influence on surface accuracy.

\section{SUMMARY}

\subsection{Conclusion}

Using industrial robots in forming tools manufacturing can be a promising approach to boost efficiency and flexibility in industrial machining applications. However, the comparably high compliance of industrial robots impairs surface accuracy and therefore hampers their usage. By conducting preliminary experiments, suitable process parameters have been identified which are used to machine a reference workpiece with a conventional toolpath strategy that could also be used on ordinary 3-axis machine tools.

The analysis of the conventional strategy's machining results has indicated that steep areas of the machined workpiece cause the tool's bending moment to oscillate which is expected to be avoided through a dynamic inclination of the tool that follows the workpiece's surface. Moreover, small circular movements with ball-nose endmills lead to low relative velocities between tooltip and workpiece and are hard to be performed by the robot leading to the hypothesis that the avoidance of circular movements increases surface accuracy.

Based on those findings, new toolpath strategies are proposed and machined. However, those new strategies are not able to compete with the conventional strategy in terms of surface accuracy. This is attributed to more complex robot movements that are required for the proposed strategies, requiring multiple axes to perform strong movements concurrently and leading to disadvantageous velocity and tool orientation progressions.

\subsection{Recommended actions}

Even though the proposed strategies have not been able to outperform the conventional strategy, the machining results have provided valuable insights from which the following recommended actions can be derived in order to improve surface accuracy:

- Toolpaths should be chosen in a way that they do not require many axes to perform large movements concurrently. Future work could analyse if a change of the clamping position can reduce axis movements while the actual path strategy remains the same.

- Changes of tool orientation should be avoided while the tool is engaged. If a tool orientation change is unavoidable while the tool is engaged, it should be done smoothly, slowly and without impacting TCP velocity.

- Since tool deflection is a major cause for surface deviations, path planning should focus on movements that are less prone to large bending moments.

\subsection{Outlook}

Future work is supposed to investigate a compensation approach that eliminates surface deviations caused by tool deflection.

\section{ACKNOWLEDGMENTS}

This research is financially supported by the "Federal Ministry for Economic Affairs and Energy" (BMWi), the "German Federation of Industrial Research Associations" (AiF) under the project number $20520 \mathrm{~N}$ and managed by the research association "European Research Association for Sheet Metal Working" (EFB).

\section{REFERENCES}

[Bisu 2011] Bisu, C., Cherif, M., Gerard, A. and K'nevez, J.Y. Dynamic Behavior Analysis for a Six Axis Industrial Machining Robot. Advanced Materials Research, 2011, No. 423, pp. 65-76. ISSN 1662-8985.

[Coelho 2011] Coelho, R.T., Rodella, H.H., Martins, V.F. and Barba J., R. An Investigation into the Use of Industrial Robots for Machining Soft and Low Density Materials with HSM Technique. Journal of the Brazilian Society of Mechanical Sciences and Engineering, 2011, Vol. 33, No. 3, pp. 343-350. ISSN 1678-5878.

[Cordes 2017] Cordes, M. and Hintze, W. Offline simulation of path deviation due to joint compliance and hysteresis for robot machining. The International Journal of Advanced Manufacturing Technology, 2017, Vol. 90, 1-4, pp. 10751083. ISSN 0268-3768.

[Hao 2019] Dynamic Behavior Analysis and Multi-sensor Modal Information Fusion for Robotic Milling System. In: $\mathrm{H}$. Yu; J. Liu; L. Liu; Z. Ju; Y. Liu; D. Zhou, eds. Intelligent Robotics and Applications. Cham: Springer International Publishing. 2019, pp. 469-481. ISBN 978-3-030-27528-0.

[IFR 2020] Executive Summary World Robotics 2020: Robot installations 2019: Global economic downturn and trade tensions leave their marks.

[Karim 2018a] Correlation between the dynamic behavior of a six-axis industrial robot and the milling process. In: A. Karim; M. Munz; A. Verl, eds. ISR 2018: $50^{\text {th }}$ International Symposium on Robotics: 20-21 June 2018. Frankfurt am Main: VDE. 2018. ISBN 3800746999.

[Karim 2018b] Karim, A., Schmid, S. and Verl, A. Pose and Feed-Direction Dependency Analysis for Milling Tasks with Industrial Robots. DEStech Transactions on Engineering and Technology Research, 2018, pp. 555-561.

[Li 2017] Li, J., Li, B., Shen, N., Qian, H. and Guo, Z. Effect of the cutter path and the workpiece clamping position on the stability of the robotic milling system. The International Journal of Advanced Manufacturing Technology, 2017, Vol. 89, 9-12, pp. 2919-2933. ISSN 0268-3768.

[Mejri 2016] Mejri, S., Gagnol, V., Le, T.-P., Sabourin, L., Ray, P. and Paultre, P. Dynamic characterization of machining robot and stability analysis. The International Journal of Advanced Manufacturing Technology, 2016, Vol. 82, 1-4, pp. 351-359. ISSN 0268-3768.

[Schmid 2017] Werkzeugmaschinen: Aufbau, Konstruktion und Systemverhalten. Haan-Gruiten: Verlag EuropaLehrmittel Nourney Vollmer GmbH \& Co. KG, 2017. ISBN 9783808550175. 